\title{
New Methods for Computing Inferences in First Order Logic *
}

\author{
J. N. Hooker \\ GSIA, Carnegie Mellon University \\ Pittsburgh, PA 15213 USA \\ April 1991 \\ Revised April 1992
}

\begin{abstract}
Recent improvements in satisfiability algorithms for propositional logic have made partial instantiation methods for first order predicate logic computationally more attractive. Two such methods have been proposed, one by R. Jeroslow and a hypergraph method for datalog formulas by G. Gallo and G. Rago. We show that they are instances of two general approaches to partial instantiation, and we develop these approaches for a large decidable fragment of first order logic (the $\exists \forall$ fragment).
\end{abstract}

\section{Introduction}

The last few years have seen a surge of interest in applying the computational methods of combinatorial optimization to logical inference problems. Most of this effort has been directed toward propositional logic $[2,3,4,5,10$, $14,15,16,17,18,19,22][23,26]$ and probabilistic logic $[1,7,12,13,20$, $24,25]$. Less work in this area has focused on predicate logic, but it is nonetheless reaching a stage at which it can make a significant contribution to computational methods.

The pathbreaking work was done by R. Jeroslow [21], who developed a partial instantiation method that reduces a satisfiability problem in first

\footnotetext{
*Working Paper 1991-11. Supported in part by the Air Force Office of Scientific Research, Grant number AFOSR-87-0292.
} 
order logic (which is equivalent to an inference problem) to a series of satisfiability problems in propositional logic. Optimization methods can then be applied to solving the latter problems. Shortly thereafter, G. Gallo and G. Rago [9] showed how to use hypergraphs to effect a partial instantiation method for "datalog" formulas, which are universally quantified Horn formulas in predicate logic.

We will show that the work of Jeroslow on the one hand, and Gallo and Rago on the other, are actually instances of two general strategies for solving inference problems by partial instantiation. Roughly speaking, Jeroslow's approach begins with a "weak" version of the problem that has a satisfying solution and strengthens it by instantiating variables until no solution exists, or until the problem is proved satisfiable. By contrast, Gallo and Rago's method begins with a "strong" version of the problem that is unsatisfiable and weakens it by instantiating variables until a solution exists or until the problem is proved unsatisfiable. The former method might be called a "primal" method and the latter a "dual" method, in analogy with linear programming, in which primal methods begin with a feasible solution and dual methods with an infeasible solution.

We will apply both approaches to solving satisfiability problems in a large but decidable fragment of first order logic (namely, the " $\exists \forall$ " fragment). Our primal method will be similar to Jeroslow's, except that we will first convert formulas to clausal form (i.e., disjunctive normal form), which considerably simplifies Jeroslow's approach. Our dual method will implement the general strategy of Gallo and Rago's hypergraph approach but for a much larger fragment of first order logic. It will not use hypergraphs, but we will indicate how their approach is a specialization of ours.

Key to the efficiency of these methods is the availability of a fast satisfiability algorithm for propositional logic, and in particular a fast algorithm for re-solving a problem after a new clause has been added (the incremental satisfiability problem). Fortunately, the research cited above has yielded enormous speedups in satisfiability algorithms in the last few years. In particular, we show elsewhere [18] how to adapt one of the new methods, a variation of the Davis-Putnam-Loveland algorithm, to exploit the information gained in the solution of the original problem to accelerate the solution of the incremented problem. For most of the problems tested, this algorithm can re-solve a satisfiability problem, after adding a clause, in only a small fraction of the time it would take to solve the incremented problem from scratch.

We begin below with a brief introduction to some concepts of propo- 
sitional and predicate logic we will need (see [11] for a fuller treatment). We then describe the primal and dual approaches to solving satisfiability problems by partial instantiation.

\section{Logical Fundamentals}

\subsection{Propositional Logic}

The formulas of propositional logic contain unanalyzed atomic propositions or variables, each of which can be true or false. Variables are joined with various logical connectives, such as $\vee$ (or), $\wedge$ (and), $\neg$ (not), $\supset$ (material implication) and $\equiv$ (equivalence, or mutual implication). The truth value of a formula depends entirely on the truth values of its atoms. For instance, if variables $x$ and $y$ are both true, then the following formula is false,

$$
(\neg x \wedge y) \vee(x \supset \neg y),
$$

since $\neg x \wedge y$ is false when $x$ is true, and the implication $x \supset \neg y$ ("if $x$ then not- $y$ ") is false when the antecedent $x$ is true and the consequent $\neg y$ is false.

A formula $A$ is satisfiable when it is true for some assignment of values to its atomic propositions. $A$ implies $B$ when $A \wedge \neg B$ is unsatisfiable.

Formulas may be manipulated using the following rules, where $A, B$ and $C$ represent any formulas.

$$
\begin{aligned}
\neg \neg A & \equiv A \\
\neg(A \vee B) & \equiv \neg A \wedge \neg B \\
\neg(A \wedge B) & \equiv \neg A \vee \neg B \\
A \wedge(B \vee C) & \equiv(A \wedge B) \vee(A \wedge C) \\
A \vee(B \wedge C) & \equiv(A \vee B) \wedge(A \vee C) \\
A \supset B & \equiv \neg A \vee B \\
A \equiv B & \equiv(A \supset B) \wedge(B \supset A)
\end{aligned}
$$

A literal is a variable or its negation, and a clause is a disjunction of literals, such as $x \vee \neg y$. A formula is in clausal form, or conjunctive normal form, when it is a conjunction of clauses. Rules (2) can be used to put any given formula in clausal form. A clause containing at most one positive literal is Horn.

It may not be practical to transform a long formula $F$ to clausal form using (2), since the result can grow exponentially with the length of $F$. 
There is, however, a way of using additional variables so that the clausal expression grows only linearly with the length of $F$. First use rules (2) to remove all occurrences of $\supset$ and $\equiv$, and to move $\neg$ 's inside so that they are all attached to atomic propositions. Then $F$ is a conjunction of one or more subformulas. Consider any subformula that is not already a clause. It is a disjunction $\bigvee_{i} A_{i}$ of one or more smaller formulas $A_{i}$, where each $A_{i}$ has the form,

$$
A_{i}=\bigwedge_{j \in J_{i}} B_{i j}
$$

We can rewrite $F$ by a) introducing a new variable $z_{i}$ to represent each $A_{i}$ that is not a literal, b) encoding $z_{i} \equiv A_{i}$ in clausal form, and $\mathrm{c}$ ) asserting $\bigvee_{i} z_{i}$. We therefore replace $\bigvee_{i} A_{i}$ with the conjunction,

$$
\left(\bigvee_{i \in I_{1}} A_{i} \vee \bigvee_{i \in I_{2}} z_{i}\right) \wedge \bigwedge_{i \in I_{2}}\left(z_{i} \vee \bigvee_{j \in J_{i}} \neg B_{i j}\right) \wedge \bigwedge_{i \in I_{2}} \bigwedge_{j \in J_{i}}\left(\neg z_{i} \vee B_{i j}\right)
$$

where $I_{1}$ contains the indices of all $A_{i}$ 's that are literals and $I_{2}$ the indices of the remaining $A_{i}$ 's. We apply the same procedure to all the nonclausal conjuncts of $F$ to obtain a new $F$. We then process the new $F$ in the same way, and continue in this fashion until clausal form is obtained.

To put (1) in clausal form, for instance, we write it as $(\neg x \wedge y) \vee \neg x \vee \neg y$ and introduce a new variable $z_{1}$ for the first disjunct. This yields,

$$
\left(\neg x \vee \neg y \vee z_{1}\right) \wedge\left(z_{1} \vee x \vee \neg y\right) \wedge\left(\neg z_{1} \vee \neg x\right) \wedge\left(\neg z_{1} \vee \neg y\right)
$$

Since every conjunct is a clause, we have clausal form.

\subsection{Predicate Logic}

First order predicate logic is more versatile than propositional logic because it includes properties and relations, which are denoted by predicates. In the formula,

$$
P(a) \supset Q(a, b)
$$

the constants $a$ and $b$ denote individuals, $P$ is a 1 -place predicate, and $Q$ is a 2-place predicate. If $P(a)$ means that $a$ is a father and $Q(a, b)$ means that $a$ is the father of $b$, then (4) means that if $a$ is a father then he is the father of $b$.

Predicate logic is also called quantified logic because it contains the universal quantifier $\forall$, which means "for every," and the existential quantifier $\exists$, which means "there exists." The formula,

$$
\forall x(P(x) \supset \exists y Q(x, y)),
$$


says that for everything $x$ that is a father, there is something $y$ of which it is the father. Here $x$ is a variable that is bound by the quantifier $\forall$, and $y$ is bound by $\exists$. Unbound variables are said to be free. $Q(a, b)$ is a complete instantiation of $Q(x, y)$ because both variables $x, y$ are replaced with constants. A formula in which all predicates are completely instantiated is said to be at ground level. Finally, the logic is first order because only individuals and not predicates can be bound by quantifiers.

\subsection{Models}

To define truth and falsehood of formulas, we must introduce models. In predicate logic a model specifies the domain (the set of constants that can instantiate variables) as well as which ground level predicates are true. For instance, a model $M$ might say that $\{a, b\}$ is the domain, $P(a)$ and $P(b)$ are the only true complete instantiations of $P(x)$, and $Q(a, b)$ and $Q(b, c)$ are the only true complete instantiations of $Q(x, y)$.

Clearly (5) is true in $M$. Also (5) is satisfiable because it is true in at least one model.

The versatility of predicate logic exacts a price, part of which is that the problem of determining satisfiability is not only hard but insoluble in general. In propositional logic there is a finite procedure for checking whether a formula is satisfiable: just try all assignments of truth values to the atomic propositions. But Church [8] proved in 1936 that first order predicate logic is undecidable, meaning that there is no such procedure that works for every formula.

\section{$2.4 \quad$ Fragments}

Due to the undecidability of full first order logic, it is common to work within decidable subsets or "fragments." The various fragments are distinguished by the order of the quantifiers when formulas are converted to "prenex form," which we now define.

A formula is in prenex form if it is quantifier-free (contains no quantifiers) or all of its quantifiers precede everything else. For instance, prenex form for formula (5) is $\forall x \exists y(P(x) \supset Q(x, y))$. The part of the formula following the quantifiers is the matrix. The following rules, together with (2), suffice to transform any formula to prenex form.

$$
\exists x \neg A \equiv \neg \forall x A
$$




$$
\begin{aligned}
\forall x \neg A & \equiv \neg \exists x A \\
(\forall x A \vee B) & \equiv \forall x(A \vee B) \\
(\forall x A \wedge B) & \equiv \forall x(A \wedge B) \\
(\exists x A \vee B) & \equiv \exists x(A \vee B) \\
(\exists x A \wedge B) & \equiv \exists x(a \wedge B),
\end{aligned}
$$

where $x$ does not occur free in $B$, plus,

$$
\begin{aligned}
& (\forall x A \wedge \forall x B) \equiv \forall x(A \wedge B) \\
& (\exists x A \wedge \exists x B) \equiv \exists x(A \wedge B),
\end{aligned}
$$

and finally

$$
\forall x A \equiv \forall y A^{\prime}
$$

where $y$ does not occur free in $A$, and $A^{\prime}$ is $A$ with every occurrence of $x$ replaced by $y$. The last transformation is used to standardize variables apart.

The $\exists$ or existential fragment. Here formulas converted to prenex form contain only existential quantifiers, as does,

$$
\exists x \exists y(P(a, x) \wedge \neg P(y, b)) .
$$

In general, the prenex form is $\exists x B(x)$, where $x=\left(x_{1}, \ldots, x_{n}\right)$ is a vector of variables, $\exists x$ is short for $\exists x_{1} \ldots \exists x_{n}$, and $B(x)$ is quantifier-free.

The $\forall$ fragment. Here formulas converted to prenex form contain only universal quantifiers and so have the form $\forall x B(x)$, as does,

$$
\forall x \forall y(P(a, x) \wedge \neg P(b, y)) .
$$

When the matrix of a formula in the $\forall$ fragment is a conjunction of Horn clauses, the formula is a datalog formula.

The $\exists \forall$ fragment. Here formulas converted to prenex form may contain both existential and universal quantifiers, and all of the former precede all of the latter. Thus formulas have the form $\exists x \forall y B(x, y)$. Consider the somewhat more complex example,

$$
\neg\{\forall z \exists y[\exists x(\neg P(x, y) \wedge \neg P(z, x))] \vee \forall z P(y, z)\} .
$$

We first convert to prenex form. Bringing the first $\neg$ inside, we have,

$$
\exists z \forall y[\forall x(P(x, y) \vee P(z, x))] \wedge \exists z \neg P(y, z) .
$$


Since two quantifiers bind the variable $z$, we must standardize variables apart by renaming the $z$ 's bound by one of the quantifiers. In general, every quantifier should bind a different variable.

$$
\exists z \forall y[\forall x(P(x, y) \vee P(z, x))] \wedge \exists w \neg P(y, w)
$$

We can now bring the quantifiers to the front,

$$
\exists z \exists w \forall x \forall y[(P(x, y) \vee P(z, x)) \wedge \neg P(y, w)],
$$

to obtain prenex form. The formula is clearly in the $\exists \forall$ fragment. Note also that the matrix is in clausal form.

\section{Instantiation Methods}

\subsection{Complete Instantiation}

The basic idea of an instantiation method is to transform a given formula to a ground level formula that is satisfiable if and only if the original formula is. Optimization methods can then be used to check whether the ground level formula is satisfiable.

We begin by testing for satisfiability a formula in the $\exists$ fragment, namely (7). If (7) is satisfiable, we should be able to replace $x$ and $y$ with distinct constants and get a ground level formula that is satisfiable. Indeed if we replace $(x, y)$ with Skolem constants $(c, d)$, we get,

$$
P(a, c) \wedge \neg P(d, b),
$$

which is satisfiable (when $P(a, c)$ and $P(d, b)$ are treated as two atomic propositions). Therefore ( 7 ) is satisfiable.

We next examine a formula in the $\forall$ fragment, namely (8). If (8) is satisfiable, then it is true in some model, and it is not hard to see that it must in particular be true in some model with domain $\{a, b\}$. But for this domain (8) is equivalent to the ground level formula,

$$
\begin{aligned}
& (P(a, a) \wedge \neg P(b, a)) \wedge(P(a, b) \wedge \neg P(b, a)) \wedge \\
& (P(a, a) \wedge \neg P(b, b)) \wedge(P(a, b) \wedge \neg P(b, b)) .
\end{aligned}
$$

Since (11) is satisfiable, so is (8).

Unfortunately, a completely instantiated ground level equivalent like (11) can become impossibly long when there are more than a few constants in the original formula. 


\subsection{Partial Instantiation}

To avoid complete instantiation, we will work with partial instantiations of a formula (i.e., formulas in which not all of the variables are replaced by constants). Consider again formula (8). Rather than instantiate both $x$ and $y$ as in (11), we will begin by instantiating neither, and consider the matrix:

$$
\begin{array}{cc}
P(a, x) \wedge \neg P(b, y) \\
T
\end{array} \quad F
$$

We now treat each predicate in (12) as an atomic proposition and try to solve the resulting propositional satisfiability problem. In this case there is a solution, indicated by the ' $T$ ' and ' $F$ ' below the atoms in (12).

We now know that (12) is satisfiable. But does this mean that the original formula (8) is satisfiable? It does, provided we can use our solution of (12) to construct a model in which (8) is true. A natural way of doing so is the following: let a ground level predicate be true when it instantiates a true predicate that occurs posited in (12) and false when it instantiates a false predicate that occurs negated. Thus we make $P(a, a)$ and $P(a, b)$ true, since they instantiate the true predicate $P(a, x)$, and we make $P(b, a)$ and $P(b, b)$ false, since they instantiate the false predicate $P(b, y)$. Formula (8) is clearly true in a such model. Each of the four conjuncts in (11) is satisfied because the predicates in them inherit the same truth values that satisfy (12). We have therefore shown that (8) is satisfiable.

Since the matrix is in clausal form, we need not specify the truth value of a ground level predicate when it instantiates only a negated true predicate or a posited false predicate. For instance, if the matrix is,

$$
\begin{gathered}
P(a, x) \vee \neg P(b, y) \vee Q(a), \\
F \quad T \quad T
\end{gathered}
$$

with the truth valuation shown, the falsehood of $P(a, x)$ and the truth of $P(b, y)$ are not needed to satisfy the formula. Thus the ground level equivalent is satisfied regardless of the truth values given $P(a, a), P(a, b), P(b, a)$ and $P(b, b)$.

Our strategy, then, is to extend a truth valuation for partially instantiated predicates to a valuation for ground level predicates. This approach can break down, however, if a ground level predicate instantiates both a true posited predicate and a false negated predicate.

This happens in the case of formula (10). The matrix, which we call $B_{1}$, 
has the satisfying solution shown below.

$$
B_{1}=\begin{gathered}
(P(x, y) \vee P(a, x)) \wedge \neg P(y, b) . \\
T
\end{gathered} T \text {. }
$$

The ground level equivalent is,

$$
\begin{aligned}
& (P(a, a) \vee P(a, a)) \wedge \neg P(a, b) \wedge \\
& (P(a, b) \vee P(a, a)) \wedge \neg P(b, b) \wedge \\
& (P(b, a) \vee P(a, b)) \wedge \neg P(a, b) \wedge \\
& (P(b, b) \vee P(a, b)) \wedge \neg P(b, b) .
\end{aligned}
$$

Each of the four conjuncts (14)-(17) would be satisfied if its predicates inherited their truth values from those shown in (13). But this cannot happen, because it would make $P(a, b)$ and $P(b, b)$ both true and false. Each instantiates a true posited predicate and a false negated predicate. In $\mathrm{R}$. Jeroslow's terminology, the valuation in (13) is blocked. To show (10) is satisfiable, then, we must find an unblocked satisfying valuation of the partially instantiated formula.

(Since Jeroslow does not restrict himself to matrices in clausal form, he must consider a valuation blocked when a ground level predicate instantiates both a true and a false predicate, regardless of whether they are negated or posited. This results in considerably more blockages.)

\subsection{A Primal Approach to Avoiding Blockage}

Two ways to find unblocked valuations are the "primal" and "dual" approaches we mentioned earlier. Let us first consider the primal approach.

Blockage occurs when a true posited predicate and a false negated predicate, such as $P(x, y)$ and $P(y, b)$ in $(13)$, can be instantiated to yield the same predicate. Such an instantiation, $P(a, b)$ for instance, is called a unifier of $P(x, y)$ and $P(y, b)$. In fact there is a most general unifier of $P(x, y)$ and $P(y, b)$, in this case $P(y, b)$, and all of its instantiations receive contradictory truth values. (We can regard $P(y, b)$ as a partial instantiation of $P(x, y)$ despite the change of variable from $x$ to $y$.) We will try to avoid the contradiction by explicitly assigning $P(y, b)$ a truth value that satisfies the partially instantiated formula, and letting the instantiations of $P(y, b)$ inherit their truth value directly from $P(y, b)$.

We do this as follows. Note that the substitution $y=b$ obtains the most general unifier $P(y, b)$ from $P(x, y)$; no substitution is needed to obtain it 
from $P(y, b)$. We therefore conjoin $B_{1}$ with a formula $B_{2}$ obtained by making the substitution $y=b$ in $B_{1}$.

$$
\begin{gathered}
B_{2}= \\
F \quad P(x, b) \vee P(a, x)) \wedge \neg P(b, b) .
\end{gathered}
$$

We now check whether $B_{1} \wedge B_{2}$ is satisfiable. Since $B_{1}$ is satisfiable, we can do this in practice by adding the clauses of $B_{2}$ one at a time and using an incremental satisfiability algorithm (such as [18]). In this case $B_{1} \wedge B_{2}$ has satisfying valuations, one of which is shown in (13) and (18).

We now let the two ground level formulas (15) and (17) receive their truth values from $B_{2}$, since (15) and (17) result from the substitutions $(x, y)=$ $(a, b),(b, b)$ that complete the substitution $y=b$ that transforms $B_{1}$ to $B_{2}$. (14) and (16) still receive their values from $B_{1}$. Jeroslow says that $B_{2}$ directly covers the substitution $y=b$ and its completions $(x, y)=(a, b),(b, b) . B_{1}$ covers all substitutions, but it directly covers only those not directly covered by $B_{2}$. ( $B_{1}$ directly covers a substitution only if it directly covers all its completions.) Thus a ground level predicate receives its truth value from the $B_{k}$ that directly covers the substitution that produces the predicate.

Note that $P(y, b)$ and $P(x, b)$ have the same truth value in the solution shown in (13) and (18). This is necessary, because they are variants, which means that they differ only by a change of variable names. Thus when solving satisfiability problems, we always seek a variant-independent solution, meaning that variants are treated as the same atomic proposition.

We must now check whether the valuation in (13) and (18) is blocked. Again the differently-valued predicates $P(x, y)$ and $P(y, b)$ in $B_{1}$ have a unifier $P(y, b)$, but this is no longer a blockage, because $B_{1}$ no longer directly covers the substitution $y=b$ that transforms $P(x, y)$ into $P(y, b)$. We have a blockage elsewhere, however, because the negated false predicate $P(y, b)$ in $B_{1}$ and the posited true predicate $P(a, x)$ in $B_{2}$ have the unifier $P(a, b)$. Note that $B_{1}$ directly covers a substitution $y=a$ that transforms $P(y, b)$ into $P(a, b)$, and $B_{2}$ directly covers a substitution $(x, y)=(b, b)$ that transforms $P(a, x)$ into $P(a, b)$. We therefore generate $B_{3}$ by substituting $y=a$ in $B_{1}$. Since $B_{3}$ directly covers $y=a$, we can eliminate $B_{1}$, which no longer directly covers anything. We also generate $B_{4}$ by substituting $(x, y)=(b, b)$.

$$
\begin{aligned}
& B_{3}=(P(x, a) \vee P(a, x)) \wedge \neg P(a, b) . \\
& B_{4}=(P(b, b) \vee P(a, b)) \wedge \neg P(b, b) .
\end{aligned}
$$

Since $B_{2} \wedge B_{3} \wedge B_{4}$ is unsatisfiable, so is (10). 
The most general unifier of two occurrences of a predicate $P$ can be found with the following linear-time algorithm. First standardize variables apart by renaming all the variables in one occurrence so that it has no variables in common with the other occurrence. Going left to right from argument to argument of $P$, form equivalence classes by setting equal any pair of variables or constants that occur in the same position. If at any point two distinct constants are placed in the same equivalence class, there is no unifier. Otherwise, the most general unifier is obtained as follows: replace every variable in each equivalence class with the unique constant in that class, or if there are no constants, replace it with any one of the variables in the class.

We now state the primal method in general for the $\exists \forall$ fragment. Let a vector be " $x$-length" when it has the same number of components as $x$.

Step 1. Begin with the formula $\exists x \forall y B(x, y)$, where $B(x, y)$ is in clausal form. Substitute $d$ for $y$ to obtain $\forall y B(d, y)$, where $d$ is any $x$-length vector of distinct constants (Skolem constants), none of which occur in $B(x, y)$. Let $B_{1}=B(d, y)$, set $k=1$, and let $B_{1}$ directly cover all substitutions for $y$.

Step 2. If $B_{1} \wedge \ldots \wedge B_{k}$ cannot be satisfied by a variant-independent truth assignment, stop; the original formula is unsatisfiable. Otherwise find such a satisfying assignment.

Step 3 (check for blockage). Determine whether the assignment in Step 2 is blocked; i.e., find whether there is a predicate satisfying two conditions: a) one posited occurrence $R$ in $B_{i}$ is true and another, negated occurrence $S$ in $B_{j}$ is false (where possibly $i=j$ ); b) $R$ and $S$ have a most general unifier $U$, such that a substitution $\sigma_{R}$ directly covered by $B_{i}$ transforms $R$ to $U$ and a substitution $\sigma_{S}$ directly covered by $B_{j}$ transforms $S$ to $U$. If there is no blockage, stop; the original formula is satisfiable.

Step \& (unblock). Let $\bar{B}_{i}$ be the conjunction of clauses in $B_{i}$ that contain variables instantiated by $\sigma_{R}$, and similarly for $\bar{B}_{j}$. Carry out substitution $\sigma_{R}$ in $\bar{B}_{i}$ to obtain $B_{k+1}$. Let $B_{k+1}$ directly cover $\sigma_{R}$ and all its completions, and for each $B_{l}$ of which $B_{k+1}$ is an instantiation (including $B_{i}$ ), remove $\sigma_{R}$ and its completions from those substitutions directly covered by $B_{l}$. Carry out substitution $\sigma_{S}$ in $\bar{B}_{j}$ to obtain $B_{k+2}$. Let $B_{k+2}$ cover all completions of $\sigma_{S}$ not covered by $B_{k+1}$, and for each $B_{l}$ of which $B_{k+2}$ is an instantiation (including $B_{j}$ ), remove the substitutions directly covered by $B_{k+2}$ from those directly covered by $B_{l}$. Set $k=k+2$ and go to Step 2 .

Any $B_{i}$ may be deleted from the conjunction $B_{1} \wedge \ldots \wedge B_{k}$ as soon as it no longer directly covers any substitutions. Also a number of blockages may 
be discovered in Step 3 and dealt with in Step 4 during a single iteration of the algorithm.

If $B(x, y)$ is not in clausal form, as in Jeroslow's original method, the entire formulas $B_{i}$ and $B_{j}$ must be instantiated to obtain $B_{k+1}$ and $B_{k+2}$. This results in much more complex $B_{k}$ 's, which one would try to simplify as best he can. Also, as noted earlier, there are more blockages to undo.

The algorithm is finite because when $B_{i}$ and $B_{j}$ are instantiated with substitutions they directly cover, at least one clause that does not occur in $B_{1} \wedge \ldots \wedge B_{k}$ is created. Since there are finitely many ground clauses, the process must terminate. The correctness of the algorithm relies on the following theorem. It is straightforwardly proved and follows from Jeroslow's work in [21].

Theorem 1 (Jeroslow) $\exists x \forall y B(x, y)$ is satisfiable if $B_{1} \wedge \ldots \wedge B_{k}$ has an unblocked satisfying truth assignment for some $k$, and it is unsatisfiable if $B_{1} \wedge \ldots \wedge B_{k}$ is unsatisfiable for some $k$.

\subsection{A Dual Approach to Avoiding Blockage}

The primal approach proceeds in a reactive manner. It begins with a weak, partially instantiated version of the problem and instantiates predicates only to the extent necessary to avoid the blockages in the current satisfying truth assignment. The dual approach tries to anticipate blockages in advance and adds clauses that prevent them from happening. This results in an overly strong version of the problem that is very likely to be unsatisfiable. The problem is gradually weakened by instantiating predicates, until the original problem is proved satisfiable or proved unsatisfiable.

Since blockages occur when unifiable predicates receive different truth values, one obvious way to avoid blockage is to add clauses that equate the truth values of all pairs of unifiable predicates. But less sweeping approaches are available, particularly in special classes of problems, such as the datalog problems for which Gallo and Rago designed their hypergraph approach. We will show that, within the larger $\exists \forall$ fragment, blockage can be avoided roughly as follows: whenever a posited predicate $P$ and a negated predicate $Q$ can be unified, add the clause $\neg P \vee Q$ to force $Q$ to be true whenever $P$ is.

We will illustrate again with formula (10). Again let $B_{1}$ be the matrix shown in (13). If $B_{1}$ were unsatisfiable, we could as before conclude that (10) is unsatisfiable. Since it is satisfiable, we ignore the solution and ward off 
blockage by conjoining with $B_{1}$ the conjunction $C$ of the following clauses:

$$
\begin{aligned}
& \neg P(x, y) \vee P(y, b) \\
& \neg P(a, x) \vee P(y, b)
\end{aligned}
$$

We generate the first clause because the posited predicate $P(x, y)$ and the negated predicate $P(y, b)$ are unifiable, and the substitution that unifies them is directly covered by $B_{1}$. The second clause is similarly generated.

We now check $B_{1} \wedge C$ for the existence of a variant-independent solution. In practice we can do this by first checking $B_{1}$ for satisfiability and, if it is satisfiable, using an incremental satisfiability algorithm (e.g. [18]) to recheck satisfiability after adding the clauses of $C$ one at a time. We stop when unsatisfiability is detected and conclude that $B_{1} \wedge \bar{C}$ is unsatisfiable, where $\bar{C}$ is a conjunction of some clauses in $C$. In the present case, $C=\bar{C}$.

We next arbitrarily select a clause in $\bar{C}$, such as the last clause $\neg P(a, x) \mathrm{V}$ $P(y, b)$ added to $\bar{C}$, and carry out the substitutions in $B_{1}$ that yield the unifier $P(a, b)$ to obtain $B_{2}$ and $B_{3}$ :

$$
\begin{aligned}
& B_{2}=(P(b, y) \vee P(a, b)) \wedge \neg P(y, b) \\
& B_{3}=(P(a, a) \vee P(a, a)) \wedge \neg P(a, b)
\end{aligned}
$$

Thus $B_{2}$ directly covers $x=b$ and $B_{3}$ directly covers $(x, y)=(a, a)$. $B_{1}$ now directly covers only $(x, y)=(a, b)$.

Since $B_{1} \wedge B_{2} \wedge B_{3}$ is satisfiable, we start generating clauses in $C$. For instance, we generate the following clause because $P(y, b)$, negated in $B_{1}$, and $P(b, y)$ in $B_{3}$ have the unifier $P(b, b)$, and because $B_{1}$ directly covers a substitution $(x, y)=(a, b)$ and $B_{3}$ a substitution $(x, y)=(b, b)$ that effects the unification.

$$
\neg P(b, y) \vee P(y, b) .
$$

In fact, if we let $\bar{C}$ consist of (21) alone, $B_{1} \wedge B_{2} \wedge B_{3} \wedge \bar{C}$ is unsatisfiable. Thus we generate $B_{4}$ and $B_{5}$ :

$$
\begin{aligned}
& B_{4}=(P(b, b) \vee P(a, b)) \wedge \neg P(b, b) \\
& B_{5}=(P(a, b) \vee P(a, a)) \wedge \neg P(b, b) .
\end{aligned}
$$

We can drop $B_{1}$ because it no longer directly covers anything. Since $B_{2} \wedge$ $B_{3} \wedge B_{4} \wedge B_{5}$ is unsatisfiable, we conclude the same for (10).

A precise statement of the algorithm follows.

Step 1. Begin with the formula $\exists x \forall y B(x, y)$, where $B(x, y)$ is in clausal form. Substitute $d$ for $y$ to obtain $\forall y B(d, y)$, where $d$ is any $x$-length vector 
of distinct Skolem constants, none of which occur in $B(x, y)$. Let $B_{1}=$ $B(d, y)$, set $k=1$, and let $B_{1}$ directly cover all substitutions for $y$.

Step 2. If $B_{1} \wedge \ldots \wedge B_{k}$ cannot be satisfied by a variant-independent truth assignment, stop; the original formula is unsatisfiable.

Step 3 (avoid blockage). Find a conjunction $\bar{C}_{k}$ of clauses such that $B_{1} \wedge \ldots \wedge B_{k} \wedge \bar{C}_{k}$ is unsatisfiable, and such that each clause in $\bar{C}_{k}$ has the form $\neg R \vee S$, where $R$ and $S$ satisfy the following conditions: a) $R$ occurs posited in some $B_{i}$ and $S$ occurs negated in $B_{j}$ (where possibly $i=j$ ); b) $R$ and $S$ have a most general unifier $U$, such that a substitution $\sigma_{R}$ directly covered by $B_{i}$ transforms $R$ to $U$ and a substitution $\sigma_{S}$ directly covered by $B_{j}$ transforms $S$ to $U$. If there is no such $\bar{C}_{k}$, stop; the original formula is satisfiable.

Step 4 (instantiate). Pick a clause $\neg R \vee S$ in $\bar{C}_{k}$, along with the associated $B_{i}, B_{j}, \sigma_{R}, \sigma_{S}$, and $U$. (For instance, one might pick the last clause added to $\bar{C}_{k}$, as suggested above.) Let $\bar{B}_{i}$ be the conjunction of clauses in $B_{i}$ that contain variables instantiated by $\sigma_{R}$, and similarly for $\bar{B}_{j}$. Carry out instantiation $\sigma_{R}$ in $\bar{B}_{i}$ to obtain $B_{k+1}$. Let $B_{k+1}$ directly cover $\sigma_{R}$ and all its completions, and for each $B_{l}$ of which $B_{k+1}$ is an instantiation (including $B_{i}$ ) remove $\sigma_{R}$ and its completions from those substitutions directly covered by $B_{l}$. Carry out substitution $\sigma_{S}$ in $\bar{B}_{j}$ to obtain $B_{k+2}$. Let $B_{k+2}$ cover all completions of $\sigma_{S}$ not covered by $B_{k+1}$, and for each $B_{l}$ of which $B_{k+2}$ is an instantiation (including $B_{j}$ ), remove all substitutions directly covered by $B_{k+2}$ from those directly covered by $B_{l}$. Set $k=k+2$ and go to Step 2 .

The algorithm terminates because it, like the primal algorithm, adds at least one new clause each iteration. The correctness of the algorithm relies on the following theorem.

Theorem 2 The formula $\exists x \forall y B(x, y)$ is satisfiable if $\bar{C}_{k}$ fails to exist for some $k$, and it is unsatisfiable if $B_{1} \wedge \ldots \wedge B_{k}$ has, for some $k$, no satisfiable variant-independent truth assignment.

Proof. The second part of the theorem follows from Theorem 1. Suppose, then, that $\bar{C}_{k}$ does not exist. Then $B_{1} \wedge \ldots \wedge B_{k} \wedge C_{k}$ is satisfiable, where $C_{k}$ is the conjunction of all clauses satisfying (a) and (b) in Step 3. Let $v$ be a satisfying solution; i.e., each predicate $R$ receives truth value $v(R)$. By Theorem 1, it suffices to show that $v$, which satisfies $B_{1} \wedge \ldots \wedge B_{k}$, is unblocked. Suppose otherwise. Then there is a posited predicate $R$ in $B_{i}$ with $v(R)=T$ and a negated predicate $S$ in $B_{j}$ with $v(S)=F$ such that $R$ and $S$ have a most general unifier $U$, obtained by a substitution $\sigma_{R}$ 
directly covered by $B_{i}$ and a substitution $\sigma_{S}$ directly covered by $B_{j}$. But by construction, $C_{k}$ contains the conjunct $\neg R \vee S$, which means that $v(R)=T$ and $v(S)=F$ cannot satisfy $B_{1} \wedge \ldots \wedge B_{k} \wedge C_{k}$. We conclude that $v$ is unblocked.

The hypergraph approach of Gallo and Rago [9] constructs, for each value of $k$, a particular type of hypergraph ("colored B-graph") whose nodes correspond to the partially instantiated predicates in $B_{1} \wedge \ldots \wedge B_{k}$. Each (Horn) clause in $B_{1} \wedge \ldots \wedge B_{k}$ corresponds to a "blue" directed hyperarc ("B-arc") that is incident to one "head" node, representing the positive literal (consequent) of the clause, and several "tail" nodes, representing the negative literals (antecedents). A clause with no consequent is given a blue B-arc whose head node is the "False" node, and one with no antecedents is given an arc whose tail is the "True" node. It is not hard to show that $B_{1} \wedge \ldots \wedge B_{k}$ is unsatisfiable precisely when there is a particular type of directed path ("B-path"), using blue B-arcs, from the True node to the False node.

In addition to the blue B-arcs, a "red" B-arc is created for each clause in $C_{k} \cdot \bar{C}_{k}$ is constructed by finding a shortest B-path, using B-arcs of both colors, from True to False, and letting $\bar{C}_{k}$ contain the arcs in this path that lie in $C_{k}$. The clause in $\bar{C}_{k}$ chosen for instantiation corresponds to a first red B-arc along the B-path from True to False. The algorithm is accelerated slightly by adding a blue arc from $R$ to $S$ whenever $R$ occurs in some clause, $S$ occurs in some other clause, and $S$ is a variant of some (partial) instantiation of $R$.

The connection between hypergraphs and the dual partial instantiation method is discussed in greater detail in [6].

\section{References}

[1] Andersen, K. A., and J. N. Hooker, Bayesian Logic, to appear in Decision Support Systems.

[2] Billionnet, A., and A. Sutter, An efficient algorithm for the 3satisfiability problem, Research report 89-13, Centre d'Études et de Recherche en Informatique, 292 rue Saint-Martin, 75141 Paris Cedex 03 (1989). 
[3] Blair, C., R. G. Jeroslow, and J. K. Lowe, Some results and experiments in programming techniques for propositional logic, Computers and Operations Research 13 (1988) 633-645.

[4] Chandru, V., and J. N. Hooker, Logical inference: A mathematical programming perspective, in S. T. Kumara, R. L. Kashyap, and A. L. Soyster (eds.), Artificial Intelligence: Manufacturing Theory and Practice (IIE, 1988) 97-120.

[5] Chandru, V., and J. N. Hooker, Extended Horn sets in propositional logic, Journal of the ACM 38 (1991) 203-221.

[6] Chandru, V., and J. N. Hooker, Optimization Methods for Logical Inference, Wiley, to appear.

[7] Cheeseman, P., A method of computing generalized Bayesian probability values for expert systems, Proceedings of the 8th International Joint Conference on Artificial Intelligence, Karlsruhe, 1983. Morgan Kaufmann (Los Altos, CA, 1983) 198-202.

[8] Church, A., A note on the Entscheidungsproblem, Journal of Symbolic Logic 1 (1936) 40-41; correction, 101-102.

[9] Gallo, G., and G. Rago, A hypergraph approach to logical inference for datalog formulae, manuscript, Dip. Informatica, Università di Pisa, Italy, 1990.

[10] Gallo, G., and G. Urbani, Algorithms for testing the satisfiability of propositional formulae, Journal of Logic Programming 7 (1989) 45-61.

[11] Genesereth, M. R., and N. J. Nilsson, Logical Foundations of Artificial Intelligence, Morgan Kaufmann (Los Altos, CA, 1987).

[12] Georgakopolous, G., D. Kavvadias and C. H. Papadimitriou, Probabilistic satisfiability, Journal of Complexity 4 (1988) 1-11.

[13] Hailperin, T., Boole's Logic and Probability, Studies in Logic and the Foundations of Mathematics v. 85, North-Holland (1976).

[14] Hooker, J. N., Resolution vs. cutting plane solution of inference problems: Some computational experience, Operations Research Letters 7 (1988) $1-7$. 
[15] Hooker, J. N., A quantitative approach to logical inference, Decision Support Systems 4 (1988) 45-69.

[16] Hooker, J. N., Generalized resolution and cutting planes, Annals of Operations Research 12 (1988) 217-239.

[17] Hooker, J. N., Input proofs and rank one cutting planes, ORSA Journal on Computing 1 (1989) 137-145.

[18] Hooker, J. N., Solving the incremental satisfiability problem, to appear in Journal of Logic Programming.

[19] Hooker, J. N. and C. Fedjki, Branch-and-cut solution of inference problems in propositional logic, Annals of Mathematics and AI 1 (1990) 123-139.

[20] Jaumard, B., P. Hansen and M. P. Aragä̈, Column generation methods for probabilistic logic, ORSA Journal on Computing 3 (1991).

[21] Jeroslow, R. E., Computation-oriented reductions of predicate to propositional logic, Decision Support Systems 4 (1988) 183-197.

[22] Jeroslow, R. E., and J. Wang, Solving propositional satisfiability problems, Annals of Mathematics and AI 1 (1990) 167-187.

[23] Kamath, A. P., N. K. Karmarkar, K. G. Ramakrishnan, and M. G. C. Resende, Computational experience with an interior point algorithm on the satisfiability problem, in R. Kannan and W. R. Pulleyblank, eds., Integer Programming and Combinatorial Optimization, University of Waterloo Press (Waterloo, Ont., 1990) 333-349.

[24] Kavvadias, D., and C. H. Papadimitriou, A linear programming approach to reasoning about probabilities, Annals of Mathematics and Artificial Intelligence 1 (1990).

[25] Nilsson, N. J., Probabilistic logic, Artificial Intelligence 28 (1986) 7187.

[26] Williams, H. P., Linear and integer programming applied to the propositional calculus, International Journal of Systems Research and Information Science 2 (1987) 81-100. 